\title{
CD44 correlates with clinicopathological characteristics and is upregulated by EGFR in breast cancer
}

\author{
HANXIAO XU ${ }^{1}$, KONGJU WU ${ }^{2}$, YIJUN TIAN ${ }^{1}$, QIAN LIU $^{1}$, NA HAN $^{1}$, \\ XUN YUAN $^{1,3}$, LU ZHANG ${ }^{1}$, GEN SHENG WU ${ }^{3}$ and KONGMING WU ${ }^{1}$ \\ ${ }^{1}$ Department of Oncology, Tongji Hospital of Tongji Medical College, Huazhong University of Science and Technology, \\ Wuhan, Hubei 430030; ${ }^{2}$ Nursing School of Pingdingshan University, Pingdingshan, Henan 467000, \\ P.R. China; ${ }^{3}$ Departments of Oncology and Pathology, Karmanos Cancer Institute, \\ Wayne State University School of Medicine, Detroit, MI 48201, USA
}

Received May 6, 2016; Accepted July 15, 2016

DOI: $10.3892 /$ ijo.2016.3639

\begin{abstract}
Cluster of differentiation 44 (CD44), a well-known transmembrane glycoprotein, serves as a promoting factor in the carcinogenesis and progression of a variety of neoplasms. Previous studies have demonstrated that aberrant expression of CD44 was associated with the initiation, invasion, metastasis, and therapy-resistance of breast cancer, but whether there was any association between CD44 and pathological characteristics of breast cancer or epidermal growth factor receptor (EGFR) has not been clearly elucidated. In this study, we utilized public microarray data analysis and tissue microarray technologies to display that CD44 level was enhanced in breast cancer and was significantly correlated with histological grade and the status of estrogen receptor, progesterone receptor and human epidermal growth factor receptor-2 (HER2) and EGFR. Furthermore, mRNA expression of CD44 in breast tumors was positively correlated with basal cytokeratin markers KRT5 and KRT17, but inversely associated with luminal marker FOXA1. Besides, Kaplan-Meier analysis showed that high $C D 44$ mRNA level had adverse impact on the progression-free survival of patients with HER2-expressing or basal-like breast
\end{abstract}

Correspondence to: Dr Kongming Wu, Department of Oncology, Tongji Hospital of Tongji Medical College, Huazhong University of Science and Technology, 1095 Jiefang Avenue, Wuhan, Hubei 430030, P.R. China

E-mail:kmwu@tjh.tjmu.edu.cn

Abbreviations: CD44, cluster of differentiation 44; EGFR, epidermal growth factor receptor; TMAs, tissue microarrays; ER, estrogen receptor; PR, progesterone receptor; HER2, human epidermal growth factor receptor-2; CSCs, cancer stem cells; HA, hyaluronic acid; PI3K, phosphoinositide 3-kinase; EMT, epithelial-mesenchymal transition; IHC, immunohistochemistry; DMEM, Dulbecco's modified Eagle's medium; FBS, fetal bovine serum; PVDF, polyvinylidene fluoride; PFS, progression-free survival; TKI, tyrosine kinase inhibitor; CD44ICD, intracellular domain of CD44

Key words: CD44, epidermal growth factor receptor, breast cancer, molecular subtypes, histological grade, prognosis cancer. Functionally, inhibition of EGFR activity by erlotinib impaired the invasion and migration ability of breast cancer cell lines. Western blot assays demonstrated that erlotinib treatment decreased the expression of CD44, accompanied with the reduced protein levels of mesenchymal and cancer stem cell markers. Collectively, this study suggested that the expression of CD44 was upregulated by EGFR pathway and CD44 had a robust impact on the development of breast cancer.

\section{Introduction}

Breast carcinoma is the most prevalent female malignance and the cause of a majority of cancer-related deaths among women worldwide (1). According to the status of estrogen receptor (ER), progesterone receptor (PR) and human epidermal growth factor 2 (Her2), breast cancer can be classified into four main distinct molecular subtypes, including luminal, Her2 overexpression, basal type and normal-like (2). Although significant achievement has been made in therapeutic strategies, the clinical outcome of breast cancer patients remains unsatisfactory due to recurrence, metastasis or chemotherapy-resistance (1). Breast cancer stem cells (CSCs), which have extraordinary ability of self-renewal, proliferation and generation of heterogenic lineages of tumor cells, account for recurrence and metastasis of breast cancer (3).

CD44, a multi-structural and multi-functional transmembrane glycoprotein, participates in the regulation of many cellular processes including cell division, adhesion and migration through binding with its main ligand the hyaluronic acid (HA) $(4,5)$. It has been demonstrated that CD44 also plays essential roles in tumorigenesis (6), invasion and metastasis, as well as therapy-response (6-8). It promotes carcinogenesis by acceleration of proliferation or suppression of apoptosis, partly resulting from stimulation of Ras-Raf-Mek-Erk-cyclin D1 signaling and phosphoinositide 3-kinase (PI3K)-Akt pathway, respectively (6). CD44, a well-known CSC marker, is involved in the complex process of epithelial-mesenchymal transition (EMT), which stimulates tumor invasion and metastasis $(5,9)$.

Epidermal growth factor receptor (EGFR), belonging to the protein kinase superfamily, plays a critical role in cell proliferation (10). It has been demonstrated that EGFR is aberrantly 
expressed in a variety of epithelial tumor types, indicating that it might be implicated in the etiology and progression of these cancers, including lung carcinoma, head and neck squamous cell carcinoma and breast cancer $(11,12)$. Positive staining of EGFR protein was observed in $\sim 45 \%$ of breast cancer (13). EGFR expression was found mainly in basal-like carcinoma, and tended to be inversely associated with hormone receptor (ER, PR) levels (13). Combined status of positive EGFR and negative ER often portended a significantly worse clinical outcome (13). In consideration of its established role in cancer cell proliferation and survival, EGFR is an effective target for cancer treatment and relative drugs against EGFR have been put into clinical use. For instance, small molecule inhibitors, such as gefitinib and erlotinib, targeted the intracellular ATP-binding site in the tyrosine kinase domain of EGFR (14-16). However, acquired resistance eventually developed in nearly all patients through second mutation of EGFR or activation of parallel signals (17). Various agents and strategies have been developed to overcome EGFR-TKIs resistance. For example, AST1306, an irreversible small molecular blocker of EGFR, HER2 and HER4, achieved promising antitumor activity even in patients previously treated with HER2 inhibitor (18).

CD44 and EGFR were both enriched in basal-like breast cancer and exerted favorable effects on breast tumor progression $(13,19)$. Therefore, we hypothesized that EGFR upregulated the expression of CD44, contributing to CSCs and mesenchymal phenotypes. To test this hypothesis, we conducted a combined analysis of available published microarray data and immunohistochemistry analysis on tissue microarrays (TMA). Furthermore, causative linkage between CD44 and EGFR in breast cancer cell lines was also investigated.

\section{Materials and methods}

Breast cancer tissue microarray and immunohistochemistry detection. To evaluate the association between CD44 protein abundance and breast cancer risks, tumor grade, as well as the status of ER, PR, Her2 and EGFR in patients with breast cancer, commercially available tissue microarray (TMA) slides (BR1502, US Biomax, Inc, Rockville, MD, USA) containing histologically confirmed tissues were purchased for immunohistochemistry (IHC) analysis. This microarray contains 150 samples including 10 normal breast tissues and 140 breast cancer samples. Due to tissue rejection, the actual number of samples enrolled was 125 including 5 normal tissues and 120 breast tumor samples. Among these 120 breast cancer samples, there were 16 grade I, 90 grade II and 14 grade III samples, and we classified grade I and grade II into low-grade tumors and grade III was high-grade. A total of 4 , 75, 24 and 17 cases were Tis, T1, T2, T3 and T4, respectively. For lymph node metastasis status, there were a total of 88 , 16, 11 and 5 tumors for N0, N1, N2 and N3, respectively. For ER status, the number of ER-negative cases was 41 and that of ER-positive ones was 79. The number of ER-negative cases was 64 and PR-positive 56. The number of HER2-negative and positive were 48 and 72, respectively. The original status of EGFR staining was displayed as '-', '+', '++' and '+++'. We classified 109 cases of EGFR stain '-' and ' + ' as low EGFR and 11 cases of '++' and '+++' into high EGFR group.
Specific primary antibody against CD44 (polyclonal rabbit antibody, 1:200; ProteinTech Group) was used for IHC with a 2-step protocol. Immunohistochemical staining was performed as previously described (20). Tissues in BR1502, embedded in paraffin, were baked at $60^{\circ} \mathrm{C}$ for $1 \mathrm{~h}$ and then deparaffinised and hydrated through a series of xylenes and alcohols. Following antigen retrieval, slides were incubated in $3 \% \mathrm{H}_{2} \mathrm{O}_{2}$ diluted in methanol for $30 \mathrm{~min}$ to block the activity of endogenous peroxidases. Non-specific binding of antibodies was blocked with $2.5 \%$ horse normal serum (20 min). Next, slides were incubated overnight with primary antibody at $4^{\circ} \mathrm{C}$. Hematoxylin was used for nuclear counterstaining.

Analysis and quantification of staining. Two evaluators, blinded to the clinical data, reviewed the immunoreactivity for CD44 protein under a light microscope. For quantification, all stains were evaluated at x200 magnifications and at least 3 areas for each core were counted. The protein expression was scored independently according to the intensity of cellular staining and the proportion of stained tumor cells. The staining intensity was scored as 0 (no staining), 1 (weak staining, light yellow), 2 (moderate staining, yellow brown) and 3 (strong staining, brown). The proportions of stained tumor cells were classified as 0 ( $\leq 5 \%$ positive cells), 1 (6-25\% positive cells), 2 (26-50\% positive cells) and 3 ( $\geq 51 \%$ positive cells). The multiplication for intensity and proportion scores was utilized to represent the level of CD44 protein abundance (21). According to the final staining score, 1-3 was grouped to low expression, while $>4$ was classified into high expression.

Cell culture and drug treatment. Breast cancer cell lines, including MDA-MB-231 and HBL100 cells, were cultured in Dulbecco's modified Eagle's medium (DMEM) with $10 \%$ fetal bovine serum (FBS, Life Technologies, Inc.). These two cell lines were seeded into two $6 \mathrm{~cm}$ culture dishes and grew to $70-80 \%$ confluence before incubation with erlotinib (Sigma) at $10 \mu \mathrm{M}$ for $24 \mathrm{~h}$. The cells were harvested for the following experiments.

Migration and invasion assay. Migration and invasion assays were performed as previously described (22). Generally, 2x10 MDA-MB-231 and HBL100 cells treated with erlotinib or vehicle control were suspended in DMEM without fetal bovine serum and seeded on an $8-\mu \mathrm{m}$ pore size Transwell filter insert (Corning Inc., Corning, NY, USA) coated with diluted Matrigel (BD Biosciences, Bedford, MA, USA). Lower chamber was filled with DMEM supplemented with $10 \%$ FBS as a chemoattractant. After incubation for $18 \mathrm{~h}$ at $37^{\circ} \mathrm{C}$, invaded cells were stained with $0.5 \%$ crystal violet solution mixed with $4 \%$ paraformaldehyde and counted by light microscopy (x200). Breast cancer cell invasion ability with or without erlotinib treatment was quantitatively measured by BD coated invasion system.

Western blot detection. Cells were washed with cold PBS, scraped into RIPA buffer and centrifuged. The cell lysates were subjected to $10 \%$ SDS-PAGE and transferred to a polyvinylidene fluoride (PVDF) hybridization transfer membrane. The primary antibodies used were as follows: CD44 (ProteinTech group), EGFR (Santa Cruz Laboratories), Snail (Cell Signaling Technology), p-EGFR (Santa Cruz 
A Normal
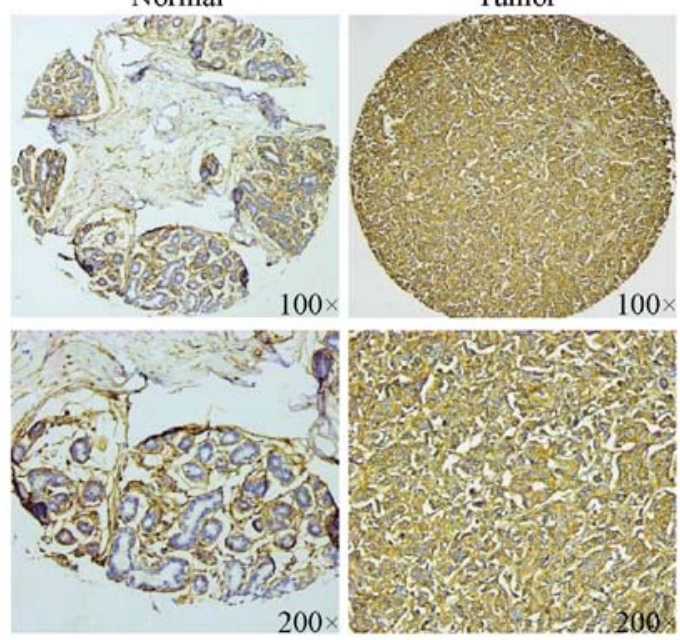

B

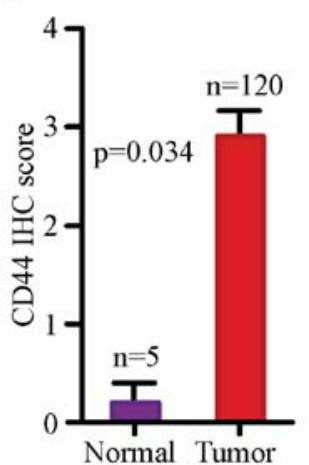

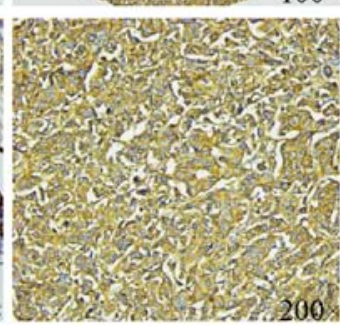

C GSE42568

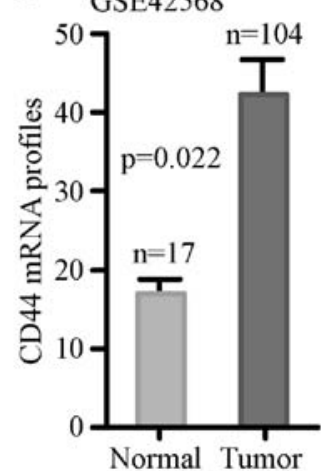

Figure 1. Analyses of CD44 in normal breast and tumor tissues. (A) Representative images of CD44 protein abundance in normal and breast tumor tissues are shown. (B) Semi-quantitative result is displayed as the mean $\pm \mathrm{SE}$. (C) Oncomine database analysis with the mRNA level of CD44 in normal and cancer tissues.

Laboratories), p-AKT (Cell Signaling Technology), p-ERK (Santa Cruz Laboratories), KLF4 (Santa Cruz Laboratories), c-Myc (Santa Cruz Laboratories), Slug (Cell Signaling Technology), Snail (Cell Signaling Technology), Vinculin (Santa Cruz Laboratories). Secondary staining and detection were carried out in accordance with standard protocols (23).

Analysis of gene expression data. GSE42568, available through the published Gene Expression Omnibus (GEO) databases and containing 17 normal cases and 104 breast cancer patients, was analyzed to evaluate the association between $C D 44$ expression and breast tumor risks, histological grade, EGFR, KRT5, KRTI7 and FOXA1. Additionally, the association between CD44 mRNA level and the progressionfree survival (PFS) of patients with HER2-overexpressing and basal-like breast cancer was analyzed on public data of KM PLOTTER.

Statistical analysis. Correlation analyses of CD44 with EGFR, KRT5, KRT6 and KRT17 were performed using SPSS 20 statistical software (SPSS Inc., Chicago, IL, USA). The Student's t-test was applied to evaluate the differences in groups as appropriate and the significance level was set at $\mathrm{p}<0.05$. The association between CD44 expression and the

A

Grade 1-2
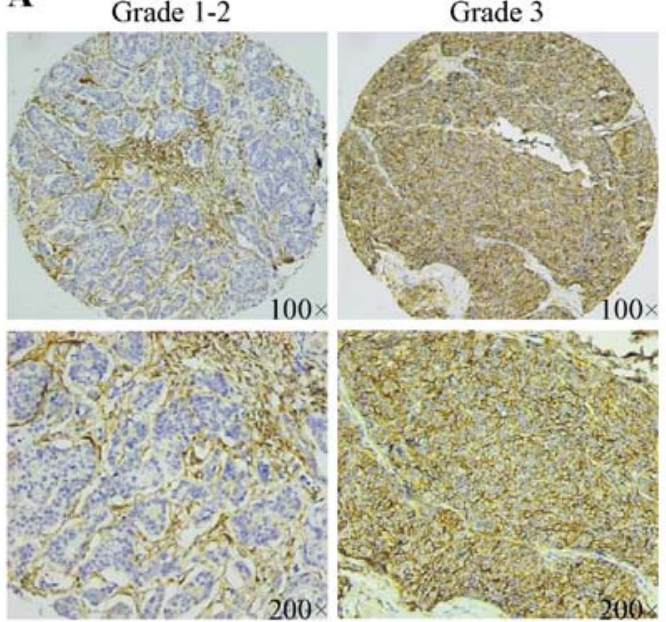

B

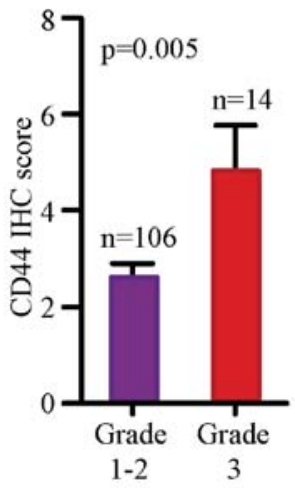

C

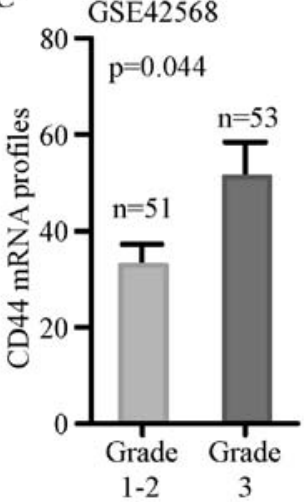

Figure 2. Analyses of CD44 in low-grade and high-grade breast tumors. (A) Representative images of CD44 protein abundance in low-grade and high-grade breast tumors are shown. (B) Semi-quantitative result is displayed as the mean \pm SE. (C) Oncomine database analysis with the mRNA level of CD44 in low-grade and high-grade breast tumors.

clinicopathological parameters was evaluated by the $\chi^{2}$ test. A two-tailed p-value of $<0.05$ was considered statistically significant. Analysis of prognosis was conducted with the Kaplan-Meier method and the log-rank test.

\section{Results}

CD44 expression is increased in breast cancer in comparison with normal breast. In order to evaluate CD44 protein level between normal breast tissues and malignant tissues, we analyzed a TMA containing 120 informative patients with breast cancer by IHC. CD44 was mainly detected on the membrane of breast cancer cells. Representative images of immunohistochemical staining for non-cancerous and cancerous tissues are shown in Fig. 1A. Next, we examined the potential association of CD44 protein abundance with breast cancer risks by using semi-quantitative criteria. The result indicated that protein abundance of CD44 was significantly higher in breast cancer tissues compared with normal tissues (Fig. 1B, $\mathrm{p}=0.034$ ).

In order to assess whether the mRNA transcription of CD44 is consistent with the protein expression, GSE42568 was assessed. The mRNA level of $C D 44$ in breast cancer was remarkably enhanced compared with normal breast tissue 
A

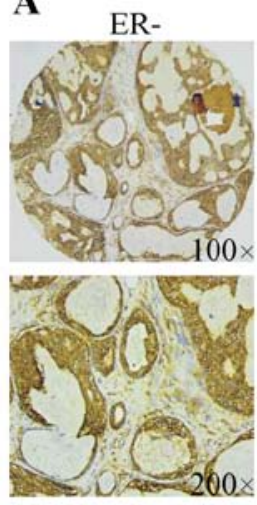

B

Luminal A $(\mathrm{p}=0.040)$

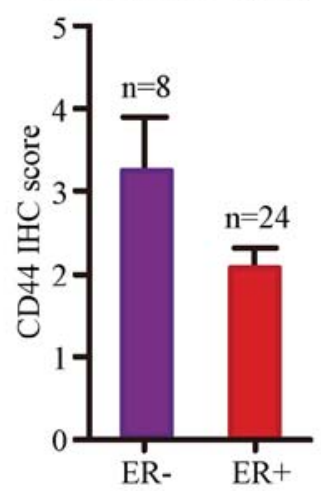

$\mathrm{ER}+$
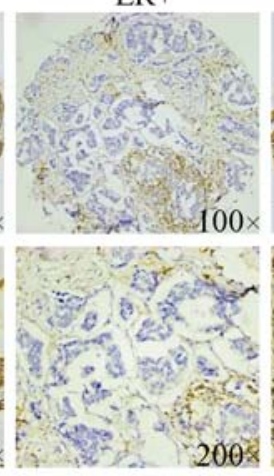

2002
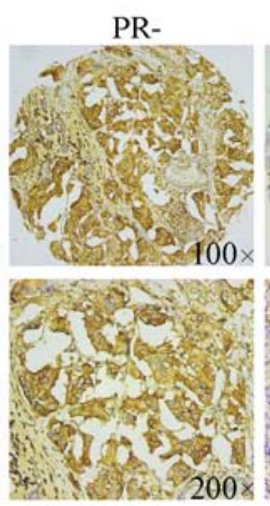

C

Luminal A $(\mathrm{p}=0.023)$

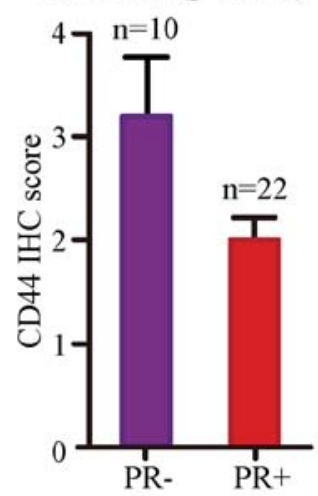

PR+
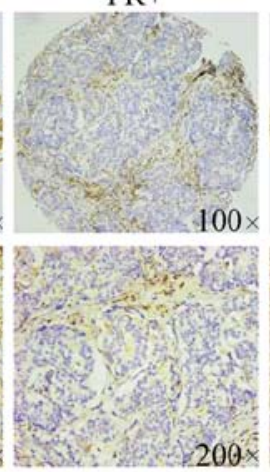

$200 \times$
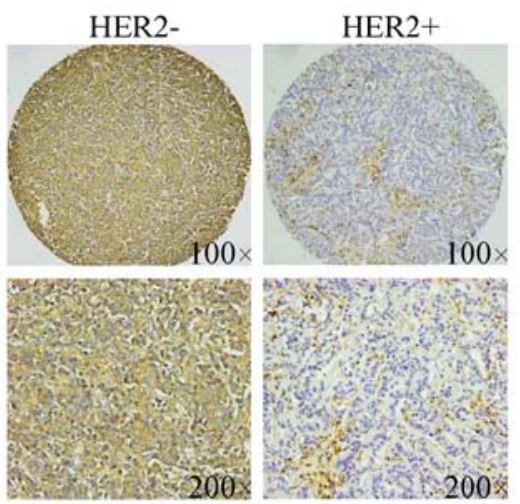

D

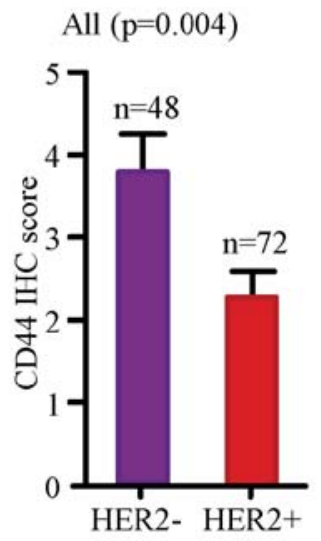

Figure 3. Analyses of CD44 with status of ER, PR and HER2. (A) Representative images of CD44 protein abundance in different status of ER, PR and HER2. (B) Semi-quantitative result of CD44 protein abundance in ER-negative and ER-positive luminal A tumors is displayed as the mean \pm SE. (C) Semiquantitative result of CD44 protein abundance in PR-negative and PR-positive luminal A tumors is displayed as the mean \pm SE. (D) Semi-quantitative result about CD44 protein abundance in HER2-negative and HER2-positive tumors is displayed as the mean \pm SE.

(Fig. 1C, $\mathrm{p}=0.022$ ). Together, our results suggested that expression of $C D 44$ was significantly upregulated at both protein and mRNA level in breast cancer tissues when compared with normal tissue.

High level of CD44 was associated with histological grade of human breast cancer. To further explore the correlation between CD44 protein abundance and histological grade, representative images of immunohistochemical staining for low-grade and high-grade cancer tissues are shown in Fig. 2A. Comparison of IHC scores suggested that CD44 protein abundance was greatly elevated in high-grade breast cancer tissues (Fig. 2B, p=0.005).

In addition, we also evaluated the mRNA expression of CD44 in both low-grade and high-grade tumors in GSE42568, and the results showed that the mRNA expression of $C D 44$ was significantly enhanced in high-grade tumors in comparison with low-grade group (Fig. 2C, p=0.044). Our results suggested that high level of $C D 44$ tended to be associated with high histological grade in breast cancer.

CD44 protein abundance tends to be associated with molecular subtype of breast cancer. To assess whether there was any association between CD44 protein abundance and the status of ER, PR and HER2, we combined IHC results with that information provided by TMA. Representative images of immunohistochemical staining for negative and positive status of ER, PR and HER2 are, respectively, shown in Fig. 3A. Statistical analysis on IHC score revealed that higher level of CD44 was significantly correlated with lower status of ER (Fig. 3B, p=0.040) and PR (Fig. 3C, p=0.023) in luminal A subtype as well as lower expression of HER2 (Fig. 3D, $\mathrm{p}=0.004)$.

Additionally, the correlation between CD44 and EGFR was analyzed. Representative images for CD44 staining in low EGFR and high EGFR samples are shown in Fig. 4A. Semiquantitative result indicated that CD44 scores were higher in high EGFR cases (Fig. 4B, p=0.004). Besides, analysis on public gene expression data indicated that $C D 44$ expression was significantly correlated with that of EGFR at mRNA level (Fig. 4C, p<0.001). Then, the correlation between CD44 mRNA level and basal cytokeratin markers KRT5 and KRT17, as well as luminal marker FOXAl were analyzed in GSE42568. The results indicated that $C D 44$ mRNA expression was positively associated with the expression of KRT5 (Fig. 4D, p=0.005) and $K R T 17$ (Fig. 4E, p=0.006), but inversely correlated with FOXAl (Fig. 4F, p<0.001).

High CD44 mRNA transcription predicts rapid progression. Public KM PLOTTER datasets were employed to evaluate the effects of $C D 44$ mRNA level on the PFS of patients with HER 2 overexpression and basal-like breast cancer. The results showed that both HER2-overexpressing (Fig. 5A, p=0.007) and basal-like patients (Fig. $5 \mathrm{~B}, \mathrm{p}=0.035$ ) with greater amount 
$\mathbf{A}$
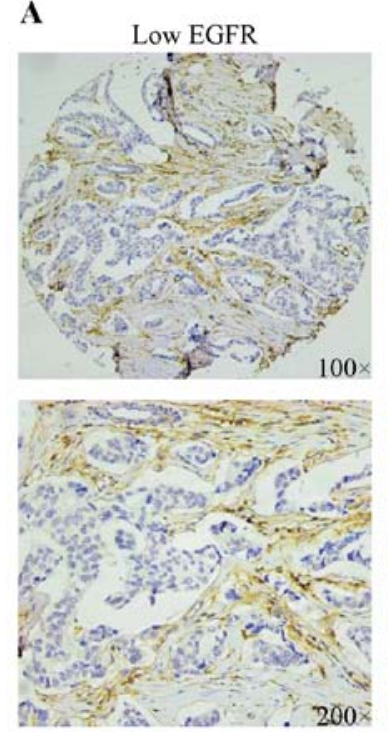

D

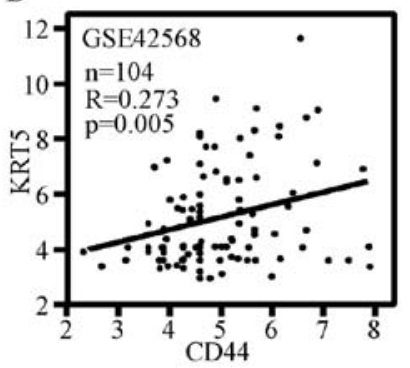

High EGFR
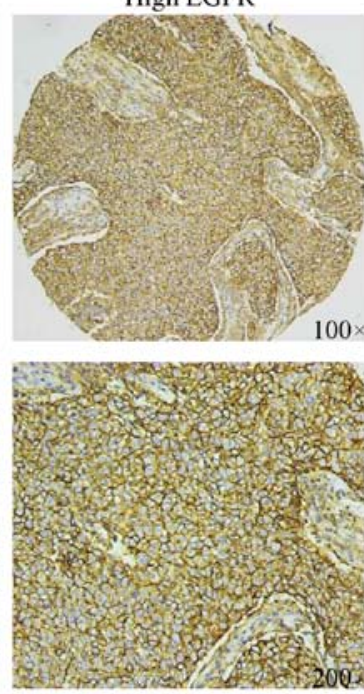

E

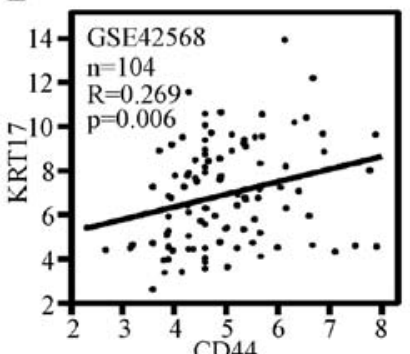

C
B
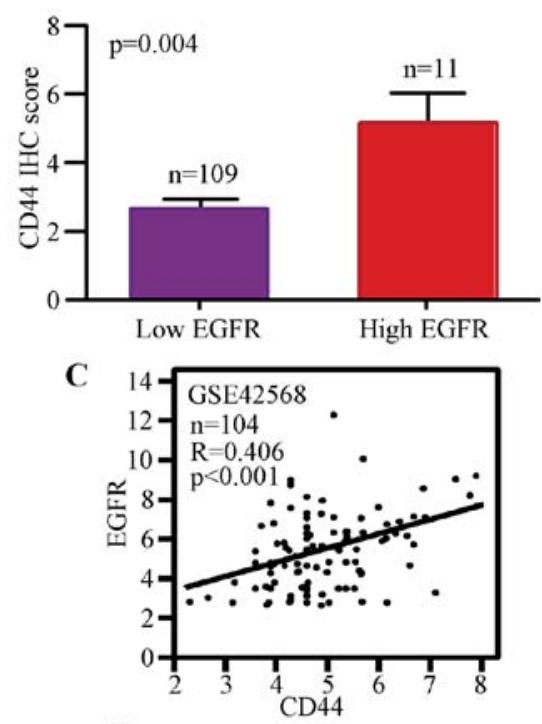

$\mathbf{F}$

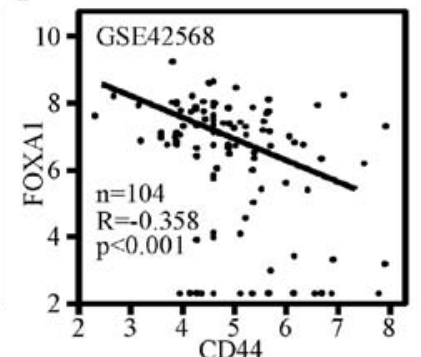

Figure 4. Analyses of CD44 in EGFR-negative and EGFR-positive breast tumors. (A) Representative images of CD44 protein abundance in EGFR low and high breast tumors are shown. (B) Semi-quantitative result of $C D 44$ protein abundance in EGFR low and high breast tumors is displayed as the mean \pm SE. Oncomine database analysis of the correlation between CD44 mRNA level and EGFR (C), KRT5 (D), KRT17 (E) and FOXA1 (F).

A

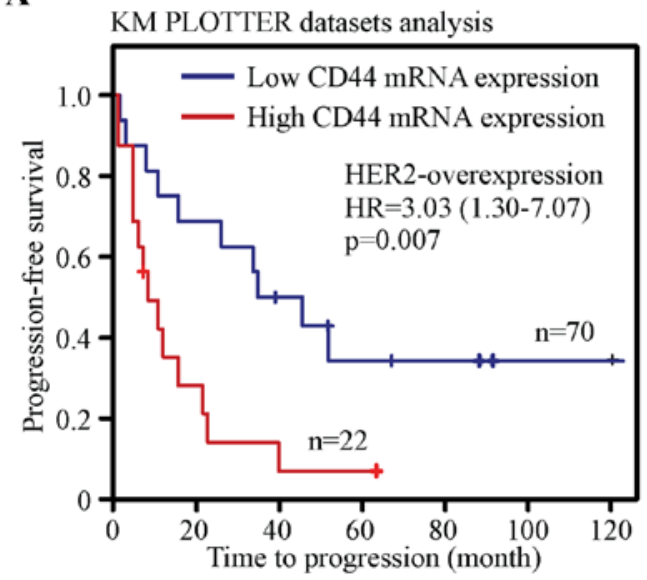

B

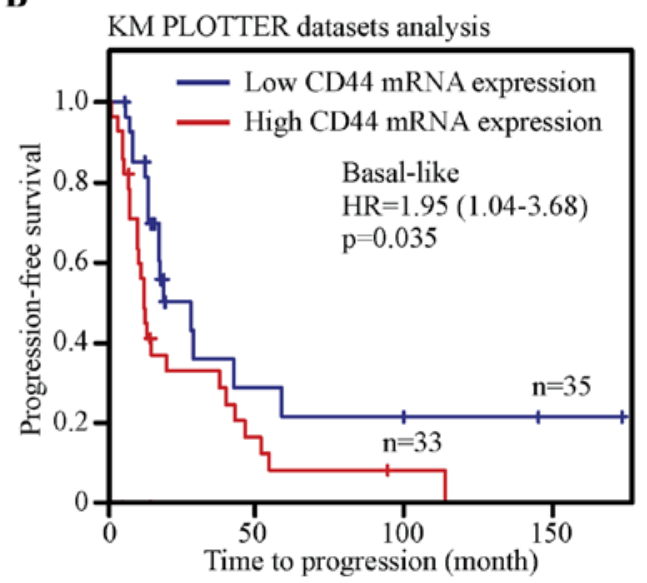

Figure 5. Kaplan-Meier survival curves for the correlation of CD44 mRNA expression with breast cancer. (A) KM PLOTTER dataset analysis showed the association between $C D 44$ mRNA expression and the PFS of patients with HER2-overexpressing breast cancer. (B) KM PLOTTER dataset analysis showed the association between CD44 mRNA expression and the PFS of patients with basal-like breast cancer.

of $C D 44$ mRNA tended to have shortened time to cancer progression, indicating that $C D 44$ exerted favorable effects on progression of patients with these two subtypes.

Inhibition of EGFR decreased invasion with reduced expression of CD44, EMT and CSC-related genes. To evaluate whether EGFR regulated CD44, a small molecular inhibitor of EGFR, erlotinib, was incubated with breast cancer cells for $24 \mathrm{~h}$, then the ability of migration and invasion was assessed. The results of transwell assay showed that inhibition of EGFR markedly reduced migration of MDA-MB-231 and HBL100 cells (Fig. 6A). Similarly, invasion capability of these two cell 
A Migration
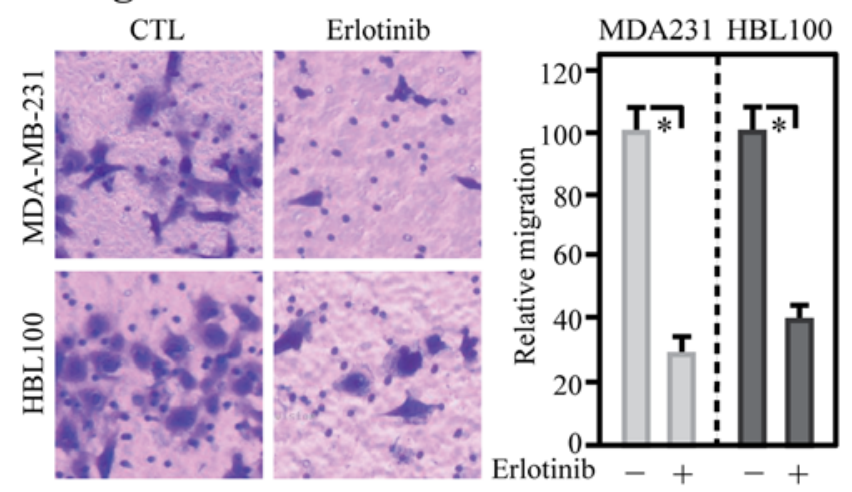

C

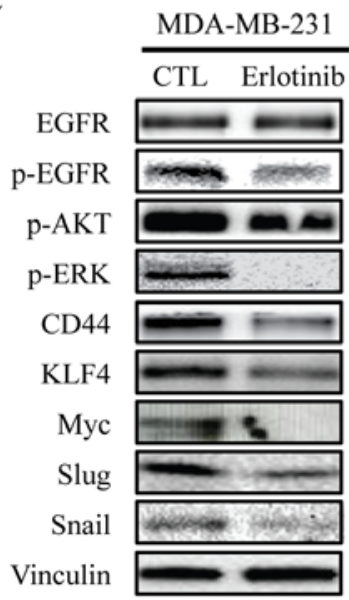

B Invasion

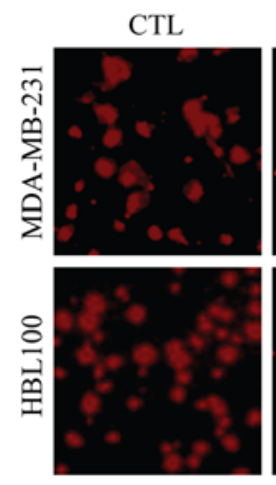

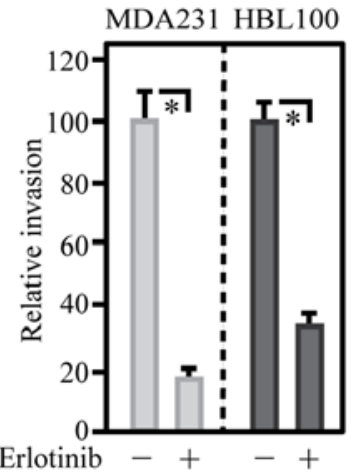

Figure 6. Inhibition of EGFR reduced migration and protein abundance of CD44, EMT and CSC-related genes. (A) Transwell assays were utilized to assess the migration. (B) Invasion ability was evaluated by an invasion kit. (C) Western blotting was employed to evaluate the protein levels of EGFR, p-EGFR, p-AKT, p-ERK, CD44, KLF4, Myc, Slug, Snail and Vinculin.

lines was also impaired by erlotinib (Fig. 6B). To detect the abundance of CD44 and related proteins after erlotinib treatment, total protein was extracted and analyzed by western blotting. The results showed that inhibition of EGFR with erlotinib in breast cancer MDA-MB-231 and HBL100 cell lines significant downregulated p-AKT, p-ERK, CD44, KLF4, Myc, Slug and Snail in these cells (Fig. 6C).

\section{Discussion}

Overwhelming number of studies have been carried out to explore the roles of CD44 in cancer. As a well-known marker of CSCs, CD44 promotes carcinogenesis of diverse tumor types, including colorectal (24), pancreatic (25) and breast cancer (26). It has been demonstrated that CD44 is a powerful regulator of EMT process as well as tumor invasion, migration and therapy-resistance (5). We provided previously the fact that CD44 was enhanced in breast cancer and closely linked to tumor grade (27). Our study further indicated that higher CD44 protein abundance tended to be parallel with the status of ER and PR, while be negatively associated with HER2 status. Finally, blockade of EGFR activity by tyrosine kinase inhibitor (TKI) induced a reduction of CD44 and a series of markers of CSCs and EMT. Thus, we drew a conclusion that CD44 served downstream of EGFR to function in the progression of breast tumor. Identification of clinically relevant genes may be explored as a molecular biomarker to aid precision therapy (28).
Consistent with previous studies, our research also offered evidence that CD44 was remarkably upregulated in breast tumor tissues in comparison with normal counterparts at both protein and mRNA levels, indicating that CD44 was involved in tumorigenesis of breast cancer. Breast cancer cells with ectopic expression of the cleaved intracellular domain of CD44 (CD44ICD) displayed potent ability of tumorigenesis and showed enhanced metastatic potential in mouse models, compared with the control cells (29). Aberrant expression and nuclear accumulation of CD44ICD played a pivotal role in transcriptional activation and nuclear localization of stemness factors such as Nanog, Sox 2 and Oct4, which are the stimulating effects of CD44 on the initiation of breast cancer (29). Our previous meta-analysis on human breast cancer tissues demonstrated that greater mRNA amount of CD44 tended to be linked to higher histological grade (27). Consistently, our immunohistochemistry analysis showed higher CD44 protein abundance was also parallel with histological grade, which provided more evidence to support that CD44 was positively associated with breast tumor grade as well as might have unfavorable impact on breast cancer cell differentiation. It is consistent with previous analysis of 448 primary breast tumors by McFarlane, et al showing that breast tumors with high CD44 protein abundance tended to be high-grade (30).

CD44, a widely recognized CSC marker and an important upregulator of EMT process, not only stimulated carcinogenesis, but also promoted metastasis and therapy-resistance (5). For instance, CD44 restrained the combination of membrane- 
associated E-cadherin and $\beta$-catenin, which promoted $\beta$-catenin to nuclear and then transcriptionally activated genes that were involved in the invasion and migration of colon cancer cells (31). Our prognostic analysis showed that high expression of CD44 mRNA had adverse impact on the PFS of patients with HER2 overexpressing and basal-like breast cancer, indicating that $C D 44$ was capable of promoting breast tumor progression. CD44 enhanced adhesion of breast cancer cells to endothelium and fibronection through $\alpha 5 \beta 1$-integrin to facilitate metastasis of breast cancer (32). Furthermore, ectopic expression of CD44 played important role in the doxorubicin-resistance of breast cancer patients, and antiCD44 mAb remarkably suppressed migration and invasion of breast cancer MCF-7 cells (33).

The status of ER, PR and HER2 were utilized to divide breast cancer into four distinct subtypes, including luminal, HER2-positive, basal-type and normal-like. Different subgroups displayed diverse biological behavior and showed different susceptibility to common therapeutics (2). Hereby, we explored whether there was any significant association between CD44 protein abundance and the status of ER, PR and HER2. Our results indicated that high content of CD44 protein abundance was negatively associated with the status of ER and PR in luminal A group and it also had an inverse linkage with HER2 status. In support, the previous research got similar conclusion that most of CD44-negative cases were observed in luminal A subtype and the number of CD44positive cases in HER2-positive samples was 4.8 times less than that of HER2-negative ones (19). KRT5 and KRT17 are well-recognized factors for basal-like breast cancer, while FOXAl was enriched in luminal type by activating ER mRNA expression (34) and maintaining ER sensitivity (35). Thus, the positive correlations between $C D 44$ with KRT5 and KRT17 as well as inverse association with FOXA1 suggested that CD44 was enriched in basal-like breast cancer, which was consistent with the finding in our meta-analysis (27).

Activated EGFR stimulated signal transduction pathways that were involved in the regulation of several cell functions such as cell proliferation and motility (36), including the Ras-ERKs (10) and PI3K-AKT pathway (37), both of which were growth-promoting signaling cascades. It has been well demonstrated that dysregulation of EGFR plays key roles in the malignant transformation and tumor development of diverse tumors such as lung cancer (38), oral cancer (39), hepatocarcinoma (40), and gastrointestinal (41), and breast cancer (42). By receptor and ligand overexpression and deficiency of specific phosphatases, EGFR promoted carcinogenesis (43). EGFR combined with DNA-PK in the nucleus to enhance DNA-PK activity and DNA repair (44), which resulted in radiotherapy-resistance.

In primary head and neck squamous cell carcinoma, the expression patterns of CD44 and EGFR were overlapping and were significantly connected (45). Although CD44 and EGFR have great impact on the initiation and progression of breast cancer, whether there was any connection between CD44 and EGFR still lacks direct evidence in breast cancer. Our study of immunohistochemistry analysis on TMA and correlation analysis on GSE42568 supported that the expression of CD44 was positively correlated with EGFR. Blocking activity of EGFR with erlotinib impaired the ability of migration and invasion of breast cancer cells. Further analysis by western blotting displayed that blockade of EGFR resulted in the downregulation of p-AKT, p-ERK and CD44. Besides, CSCs proteins KLF4, Myc and mesenchymal protein Slug and Snail were also reduced by inhibition of EGFR. These supported that high EGFR activity in basal type might drive CSC property and EMT phenotype. EMT process is controlled by both transcriptional and post-transcriptional regulation (46). The detailed mechanism by which EGFR-TKIs reverse EMT needs further classification. The EGFR-TKI reduced migration and invasion of in vitro cultured breast cancer cells. Ongoing study on xenograft mouse model will test whether EGFR-TKI inhibits in vivo invasion, thereby reducing metastasis. It has been reported that $\mathrm{CD} 44^{+} / \mathrm{CD} 24^{-}$CSCs are resistant to conventional chemotherapy, but sensitive to $\mathrm{Cdk} 2$ inhibitor (47), suggesting that patients with CD44 high expression may need personalized therapeutic strategy.

In conclusion, this study demonstrated the expression of CD44 was upregulated in breast cancer and was closely correlated with high grade. More importantly, our research indicated that EGFR exert effects on the initiation and progression of breast cancer through upregulating the level of CD44 and related markers of cancer stem cells and EMT. Identification of possible interaction between CD44 and EGFR could provide better understanding of the development of breast cancer as well as help improve therapy strategy for breast cancer patients.

\section{Acknowledgements}

This study was supported by the National Natural Science Foundation of China (NSFC) nos. 81572608 and 81172422 (K.W.), 81502209 (N.H.) and 81441087 (L.Z.).

\section{References}

1. Siegel RL, Miller KD and Jemal A: Cancer statistics, 2016. CA Cancer J Clin 66: 7-30, 2016.

2. Makki J: Diversity of breast carcinoma: Histological subtypes and clinical relevance. Clin Med Insights Pathol 8: 23-31, 2015.

3. Clarke MF, Dick JE, Dirks PB, Eaves CJ, Jamieson $\mathrm{CH}$, Jones DL, Visvader J, Weissman IL and Wahl GM: Cancer stem cells - perspectives on current status and future directions: AACR Workshop on cancer stem cells. Cancer Res 66: 9339-9344, 2006.

4. Götte M and Yip GW: Heparanase, hyaluronan, and CD44 in cancers: A breast carcinoma perspective. Cancer Res 66: 10233-10237, 2006.

5. Xu H, Tian Y, Yuan X, Wu H, Liu Q, Pestell RG and Wu K: The role of CD44 in epithelial-mesenchymal transition and cancer development. Onco Targets Ther 8: 3783-3792, 2015.

6. Zöller M: CD44: Can a cancer-initiating cell profit from an abundantly expressed molecule? Nat Rev Cancer 11: 254-267, 2011.

7. Wei X, Xu M, Wei Y, Huang F, Zhao T, Li X, Feng R and Ye BH: The addition of rituximab to CHOP therapy alters the prognostic significance of CD44 expression. J Hematol Oncol 7: $34,2014$.

8. Lv L, Liu HG, Dong SY, Yang F, Wang QX, Guo GL, Pan YF and Zhang XH: Upregulation of CD44v6 contributes to acquired chemoresistance via the modulation of autophagy in colon cancer SW480 cells. Tumour Biol: Jan 9, 2016 (Epub ahead of print).

9. Erb U, Megaptche AP, Gu X, Büchler MW and Zöller M: CD44 standard and CD44v10 isoform expression on leukemia cells distinctly influences niche embedding of hematopoietic stem cells. J Hematol Oncol 7: 29, 2014.

10. Yarden Y and Sliwkowski MX: Untangling the ErbB signalling network. Nat Rev Mol Cell Biol 2: 127-137, 2001. 
11. Veale D, Ashcroft T, Marsh C, Gibson GJ and Harris AL: Epidermal growth factor receptors in non-small cell lung cancer. Br J Cancer 55: 513-516, 1987.

12. Weichselbaum RR, Dunphy EJ, Beckett MA, Tybor AG, Moran WJ, Goldman ME, Vokes EE and Panje WR: Epidermal growth factor receptor gene amplification and expression in head and neck cancer cell lines. Head Neck 11: 437-442, 1989.

13. Klijn JG, Berns PM, Schmitz PI and Foekens JA: The clinical significance of epidermal growth factor receptor (EGF-R) in human breast cancer: A review on 5232 patients. Endocr Rev 13: 3-17, 1992.

14. Wakeling AE, Guy SP, Woodburn JR, Ashton SE, Curry BJ, Barker AJ and Gibson KH: ZD1839 (Iressa): An orally active inhibitor of epidermal growth factor signaling with potential for cancer therapy. Cancer Res 62: 5749-5754, 2002.

15. Hidalgo M, Siu LL, Nemunaitis J, Rizzo J, Hammond LA, Takimoto C, Eckhardt SG, Tolcher A, Britten CD, Denis L, et al: Phase I and pharmacologic study of OSI-774, an epidermal growth factor receptor tyrosine kinase inhibitor, in patients with advanced solid malignancies. J Clin Oncol 19: 3267-3279, 2001.

16. Sun W, Yuan X, Tian Y, Wu H, Xu H, Hu G and Wu K: Non-invasive approaches to monitor EGFR-TKI treatment in non-small-cell lung cancer. J Hematol Oncol 8: 95, 2015.

17. Niu FY and Wu YL: Novel agents and strategies for overcoming EGFR TKIs resistance. Exp Hematol Oncol 3: 2, 2014.

18. Zhang J, Cao J, Li J, Zhang Y, Chen Z, Peng W, Sun S, Zhao N, Wang J, Zhong D, et al: A phase I study of AST1306, a novel irreversible EGFR and HER2 kinase inhibitor, in patients with advanced solid tumors. J Hematol Oncol 7: 22, 2014.

19. Gudadze M, Kankava K, Mariamidze A, Mosidze T and Burkadze G: Distribution of CD44/CD24 positive cells in ductal invasive carcinoma of breast of different grade and molecular subtype. Georgian Med News 222: 50-57, 2013.

20. Liu Y, Zhou R, Yuan X, Han N, Zhou S, Xu H, Guo M, Yu S, Zhang C, Yin T, et al: DACH1 is a novel predictive and prognostic biomarker in hepatocellular carcinoma as a negative regulator of Wnt/ $\beta$-catenin signaling. Oncotarget 6: 8621-8634, 2015.

21. Xie JW, Chen PC, Zheng CH, Li P, Wang JB, Lin JX, Lu J, Chen QY, Cao LL, Lin M, et al: Evaluation of the prognostic value and functional roles of CD44v6 in gastric cancer. J Cancer Res Clin Oncol 141: 1809-1817, 2015.

22. Han N, Yuan X, Wu H, Xu H, Chu Q, Guo M, Yu S, Chen Y and $\mathrm{Wu} \mathrm{K}$ : DACH1 inhibits lung adenocarcinoma invasion and tumor growth by repressing CXCL5 signaling. Oncotarget 6: 5877-5888, 2015.

23. Chu Q, Han N, Yuan X, Nie X, Wu H, Chen Y, Guo M, Yu S and Wu K: DACH1 inhibits cyclin D1 expression, cellular proliferation and tumor growth of renal cancer cells. J Hematol Oncol 7: $73,2014$.

24. Du L, Wang H, He L, Zhang J, Ni B, Wang X, Jin H, Cahuzac N, Mehrpour M, Lu Y, et al: CD44 is of functional importance for colorectal cancer stem cells. Clin Cancer Res 14: 6751-6760, 2008.

25. Wang D, Zhu H, Liu Y, Liu Q, Xie X, Zhou Y, Zhang L, Zhu Y, Zhang $\mathrm{Z}$ and $\mathrm{Su} \mathrm{Z}$ : The low chamber pancreatic cancer cells had stem-like characteristics in modified transwell system: Is it a novel method to identify and enrich cancer stem-like cells? BioMed Res Int 2014: 760303, 2014.

26. Xie G, Yao Q, Liu Y, Du S, Liu A, Guo Z, Sun A, Ruan J, Chen L, Ye C, et al: IL-6-induced epithelial-mesenchymal transition promotes the generation of breast cancer stem-like cells analogous to mammosphere cultures. Int J Oncol 40: 1171-1179, 2012.

27. Xu H, Tian Y, Yuan X, Liu Y, Wu H, Liu Q, Wu GS and Wu K: Enrichment of CD44 in basal-type breast cancer correlates with EMT, cancer stem cell gene profile, and prognosis. Onco Targets Ther 9: 431-444, 2016.

28. Smith AD, Roda D and Yap TA: Strategies for modern biomarker and drug development in oncology. J Hematol Oncol 7: 70, 2014

29. Cho Y, Lee HW, Kang HG, Kim HY, Kim SJ and Chun KH: Cleaved CD44 intracellular domain supports activation of stemness factors and promotes tumorigenesis of breast cancer. Oncotarget 6: 8709-8721, 2015.

30. McFarlane S, Coulter JA, Tibbits P, O'Grady A, McFarlane C, Montgomery N, Hill A, McCarthy HO, Young LS, Kay EW, et al: CD44 increases the efficiency of distant metastasis of breast cancer. Oncotarget 6: 11465-11476, 2015.
31. Cho SH, Park YS, Kim HJ, Kim CH, Lim SW, Huh JW, Lee JH and Kim HR: CD44 enhances the epithelial-mesenchymal transition in association with colon cancer invasion. Int J Oncol 41: 211-218, 2012.

32. McFarlane S, McFarlane C, Montgomery N, Hill A and Waugh DJ: CD44-mediated activation of $\alpha 5 \beta 1$-integrin, cortactin and paxillin signaling underpins adhesion of basal-like breast cancer cells to endothelium and fibronectin-enriched matrices. Oncotarget 6: 36762-36773, 2015.

33. Uchino M, Kojima H, Wada K, Imada M, Onoda F, Satofuka H, Utsugi $T$ and Murakami Y: Nuclear beta-catenin and CD44 upregulation characterize invasive cell populations in nonaggressive MCF-7 breast cancer cells. BMC Cancer 10: 414, 2010.

34. Bernardo GM, Lozada KL, Miedler JD, Harburg G, Hewitt SC, Mosley JD, Godwin AK, Korach KS, Visvader JE, Kaestner KH, et al: FOXA1 is an essential determinant of ERalpha expression and mammary ductal morphogenesis. Development 137: 2045-2054, 2010.

35. Kouros-Mehr H, Slorach EM, Sternlicht MD and Werb Z: GATA-3 maintains the differentiation of the luminal cell fate in the mammary gland. Cell 127: 1041-1055, 2006.

36. Mitsudomi T and Yatabe Y: Epidermal growth factor receptor in relation to tumor development: EGFR gene and cancer. FEBS J 277: 301-308, 2010.

37. Okano J, Gaslightwala I, Birnbaum MJ, Rustgi AK and Nakagawa H: Akt/protein kinase B isoforms are differentially regulated by epidermal growth factor stimulation. J Biol Chem 275: 30934-30942, 2000.

38. Cattaneo F, Iaccio A, Guerra G, Montagnani S and Ammendola R: NADPH-oxidase-dependent reactive oxygen species mediate EGFR transactivation by FPRL1 in WKYMVm-stimulated human lung cancer cells. Free Radic Biol Med 51: 1126-1136, 2011.

39. Brusevold IJ, Tveteraas IH, Aasrum M, Ødegård J, Sandnes DL and Christoffersen T: Role of LPAR3, PKC and EGFR in LPA-induced cell migration in oral squamous carcinoma cells. BMC Cancer 14: 432, 2014

40. Tveteraas IH, Müller KM, Aasrum M, Ødegård J, Dajani $\mathrm{O}$, Guren T, Sandnes D and Christoffersen T: Mechanisms involved in PGE2-induced transactivation of the epidermal growth factor receptor in MH1C1 hepatocarcinoma cells. J Exp Clin Cancer Res 31: 72, 2012

41. Yoshida K, Fujino H, Otake S, Seira N, Regan JW and Murayama T: Induction of cyclooxygenase-2 expression by prostaglandin E2 stimulation of the prostanoid EP4 receptor via coupling to Gai and transactivation of the epidermal growth factor receptor in HCA-7 human colon cancer cells. Eur J Pharmacol 718: 408-417, 2013.

42. Zajac M, Law J, Cvetkovic DD, Pampillo M, McColl L, Pape C, Di Guglielmo GM, Postovit LM, Babwah AV and Bhattacharya M: GPR54 (KISS1R) transactivates EGFR to promote breast cancer cell invasiveness. PLoS One 6: e21599, 2011.

43. Kondapaka SB, Fridman R and Reddy KB: Epidermal growth factor and amphiregulin up-regulate matrix metalloproteinase- 9 (MMP-9) in human breast cancer cells. Int J Cancer 70: 722-726, 1997.

44. Dittmann K, Mayer C, Kehlbach R and Rodemann HP: Radiation-induced caveolin-1 associated EGFR internalization is linked with nuclear EGFR transport and activation of DNA-PK. Mol Cancer 7: 69, 2008.

45. Leinung M, Ernst B, Döring C, Wagenblast J, Tahtali A, Diensthuber M, Stöver T and Geissler C: Expression of ALDH1A1 and CD44 in primary head and neck squamous cell carcinoma and their value for carcinogenesis, tumor progression and cancer stem cell identification. Oncol Lett 10: 2289-2294, 2015.

46. Guo F, Parker Kerrigan BC, Yang D, Hu L, Shmulevich I, Sood AK, Xue F and Zhang W: Post-transcriptional regulatory network of epithelial-to-mesenchymal and mesenchymal-toepithelial transitions. J Hematol Oncol 7: 19, 2014.

47. Opyrchal M, Salisbury JL, Iankov I, Goetz MP, McCubrey J, Gambino MW, Malatino L, Puccia G, Ingle JN, Galanis E, et al: Inhibition of Cdk2 kinase activity selectively targets the $\mathrm{CD} 44^{+}$ CD24\% low stem-like subpopulation and restores chemosensitivity of SUM149PT triple-negative breast cancer cells. Int J Oncol 45: 1193-1199, 2014. 